\title{
Comparación del efecto de la terapia prolongada con antipsicóticos atípicos o convencionales en la incidencia de diabetes mellitus 2. Revisión sistemática y metaanálisis
}

\author{
Jorge Sapunar $\mathbf{Z}^{1,2,3}$, Sergio Muñoz $N^{3}$, Tatiana Vásquez $A^{2}$. \\ Risk of type 2 diabetes mellitus \\ among users of atypical antipsychotic \\ drugs or conventional treatment. \\ Systematic review
}

Atypical antipsychotic drugs have less extrapyramidal side effects and are more effective to control the clinical manifestations of schizophrenia. However, their use may be associated to a higher incidence of weight gain, dyslipidemia, metabolic syndrome, glucose intolerance and type 2 diabetes mellitus. We performed a systematic literature search to evaluate the risk of type 2 diabetes mellitus incidence associated to the use of atypical antipsychotic drugs, compared to conventional treatment. If users of all types of atypical antipsychotic drugs are compared with users of conventional treatment, no significant differences in the incidence of type 2 diabetes mellitus were observed. If individual drugs are evaluated, clozapine and risperidone are associated with a higher risk of diabetes than haloperidol. Quetiapine is associated with a lower risk of diabetes than conventional treatment. The quality of the evidence found was low, therefore new studies should be performed (Rev Méd Chile 2009; 137: 1417-26).

(Key words: Antipsychotic agents; Aripiprazole; Diabetes mellitus; Clozapine; Olanzapine; Quetiapine; Risperidone; Ziprasidone; Zotepine)

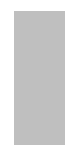

Recibido el 9 de abril, 2009. Aprobado el 6 de octubre, 2009.

${ }^{1}$ Unidad de Endocrinología, ${ }^{2}$ Departamento de Medicina Interna, Facultad de Medicina, Universidad de La Frontera. ${ }^{3}$ Centro de Excelencia CIGES, Facultad de Medicina, Universidad de La Frontera. Temuco, Chile.

L os antipsicóticos atípicos son un grupo de fármacos introducidos sucesivamente para el tratamiento de enfermedades psiquiátricas crónicas, en consideración a una menor frecuencia de

Correspondencia a: Dr. Jorge Sapunar Zenteno. Antumalal 01024, Temuco, Chile. E mail: jsapunar@ufro.cl efectos adversos extrapiramidales respecto a los llamados antipsicóticos convencionales. También tendrían una mayor efectividad en controlar las manifestaciones clínicas de la esquizofrenia. Son considerados antipsicóticos atípicos: clozapina, olanzapina, risperidona, quetiapina, zotepina, ziprasidona y aripiprazol, en tanto que clorpromazina, tioridazina, haloperidol, pimozida, 
trifluoperazina, tiotixeno, flupentixol y flufenazina son ejemplos de antipsicóticos convencionales ${ }^{1,2}$. A pesar de la mejoría en la calidad de vida de los pacientes con enfermedades psiquiátricas crónicas relacionadas con el cambio a esquemas terapéuticos con antipsicóticos atípicos, se han comunicado evidencias, no siempre de buena calidad, asociando el uso de estos fármacos con aumento ponderal $^{3,4}$, resistencia insulínica ${ }^{5-9}$, dislipidemia ${ }^{10,11}$, mayor prevalencia de síndrome metabólico, intolerancia a los hidratos de carbono y diabetes mellitus $2^{11-29}$ y el desarrollo de complicaciones agudas de la diabetes mellitus ${ }^{30}$.

La mayor parte de los efectos adversos metabólicos de los antipsicóticos atípicos fueron observados en pacientes con esquizofrenia, enfermedad con una mortalidad asociada que duplica la mortalidad general ${ }^{1}$. Susce y cols reportaron, en un estudio de corte transversal, que el riesgo de obesidad y diabetes mellitus en pacientes con enfermedades mentales crónicas, principalmente esquizofrenia, duplicaba al de la población general ${ }^{31}$. Subramaniam y cols encontraron que la prevalencia de intolerancia a los hidratos de carbono y diabetes mellitus en pacientes con esquizofrenia, sin tratamiento con antipsicóticos atípicos, fue 30,9\% y 16\%, respectivamente. Estas cifras superan largamente las observadas en la población general donde se hizo el estudio $^{32}$.

Debido a la asociación que tienen la esquizofrenia y otras enfermedades psiquiátricas crónicas con trastornos cardiometabólicos, el valor que tienen los reportes de casos clínicos y estudios de corte transversal para asociar el uso de antipsicóticos atípicos con estos desenlaces es limitado. Algunos estudios han investigado la incidencia de diabetes mellitus 2 asociada al uso de antipsicóticos atípicos en comparación con la observada en pacientes sin tratamiento o con antipsicóticos convencionales, pero su calidad metodológica en general es baja y sus resultados contradictorios $^{33-44}$.

El propósito de esta revisión sistemática es obtener la mejor evidencia disponible que permita conocer el riesgo de diabetes mellitus 2 incidente asociada al uso de antipsicóticos atípicos en comparación con el tratamiento convencional, en pacientes con enfermedades psiquiátricas crónicas.

\section{MATERIAL Y MÉTODO}

Realizamos una revisión sistemática de la literatura buscando estudios que compararan la incidencia de diabetes mellitus 2 en pacientes en tratamiento con antipsicóticos atípicos con la observada en pacientes recibiendo antipsicóticos convencionales. Se realizó una búsqueda computarizada por MEDLINE utilizando la estrategia: [atypical antipsychotics] OR clozapine OR olanzapine OR risperidone OR quetiapine OR aripiprazole AND diabetes mellitus. Adicionalmente realizamos una búsqueda manual de las referencias bibliográficas de los estudios originales.

Se consideraron elegibles revisiones sistemáticas, ensayos clínicos controlados y estudios observacionales de asociación.

Se seleccionaron para la extracción de datos y análisis aquellos estudios elegibles que incluyeran sólo pacientes sin diabetes mellitus previo a la exposición y en que el desenlace diabetes mellitus 2 incidente fuera definido explícitamente. Fue también criterio de selección que el estudio original estableciera un período mínimo de observación para atribuir el evento diabetes mellitus 2 incidente a la exposición evaluada. En todos los estudios seleccionados la condición de diabético fue establecida por el registro consultado o por estar recibiendo terapia para la enfermedad (hipoglicemiantes orales, insulina). En la mayor parte de los estudios se consideró que la diabetes mellitus incidente era atribuible a la terapia evaluada con al menos 2 meses de exposición.

La calidad de los estudios fue expresada de acuerdo a la escala de Oxford (Oxford Centre for Evidence-based Medicine Levels of Evidence, May 2001), considerando además la calidad de la fuente de obtención de datos de los pacientes y de la definición de diabetes mellitus, el tiempo requerido para atribuir a la exposición el desenlace y el reporte de otras exposiciones o variables de control como edad, género, estado nutricional y antecedentes familiares de diabetes mellitus.

Dos de los autores revisaron los estudios elegibles obtenidos en MEDLINE excluyendo aquellos que no cumplían con los criterios de selección y evaluando su calidad. De cada estudio original se obtuvo el número de eventos reportados con la exposición evaluada (antipsicóticos atípicos) y con los comparadores. El análisis de 
los datos se realizó mediante los programa RevMan 4.2 (Cochrane Iniciative) y STATA 10.0 (STATA Corp.) El riesgo del desenlace estudiado fue expresado como odds ratio (OR) en consideración a la debilidad de la evidencia disponible. La homogeneidad de los estudios originales fue expresada mediante valor $\mathrm{p}$ en un modelo de efectos aleatorios y la consistencia del análisis por $\mathrm{I}^{2}$ de Higgins.

La sensibilidad de los métodos estadísticos utilizados se evaluó comparando la magnitud de efecto utilizando OR o RR y comparando la homogeneidad mediante efectos fijos o aleatorios.

\section{Resultados}

Mediante la estrategia de búsqueda explicitada encontramos 2.492 citas bibliográficas en MEDL$\mathrm{NE}$, de las cuales 31 cumplían con los criterios de selección. Al revisar los artículos en extenso, sólo
12 de ellos fueron considerados para el análisis. No encontramos estudios que cumplieran con los criterios de selección y que evaluaran los antipsicóticos atípicos zotepina, ziprasidona y aripiprazol.

En la Tabla 1 se presentan los 19 artículos excluídos y la razón de su exclusión ${ }^{11-29}$. En algunos casos en lugar de la diabetes mellitus 2 se consideraron como desenlaces síndrome metabólico o intolerancia a los hidratos de carbono, en otros no hubo comparador o se compararon antipsicóticos atípicos entre sí.

La Tabla 2 muestra los 12 estudios incluidos en el análisis ${ }^{33-44}$. Se trata de un metaanálisis que incluyó ensayos clínicos que reportaron la diabetes mellitus 2 como efecto adverso ${ }^{35}$, un ensayo clínico aleatorizado que comparó olanzapina con amisulpiride ${ }^{43}$, un estudio de casos y controles anidado en una cohorte ${ }^{39}$, un estudio de corte transversal ${ }^{44}$ y 8 cohortes retrospectivas. En todos los estudios las definiciones de diabetes mellitus 2

Tabla 1. Estudios originales elegibles excluidos del análisis y el motivo de su exclusión

\begin{tabular}{|c|c|c|}
\hline ID Estudio & $\begin{array}{l}\text { Publicación } \\
\text { (año) }\end{array}$ & Razones de la exclusión \\
\hline Caro $^{12}$ & 2002 & Comparó antipsicóticos atípicos \\
\hline De Hert ${ }^{13}$ & 2008 & Desenlace fue síndrome metabólico (ATP III) \\
\hline Filakovic ${ }^{14}$ & 2005 & Desenlace fue intolerancia por hidratos de carbono \\
\hline Henderson ${ }^{15}$ & 2000 & No hubo comparación con otras exposiciones \\
\hline Henderson 15 & 2005 & No hubo comparación con otras exposiciones \\
\hline Koller ${ }^{16}$ & 2004 & Serie de casos de diabetes mellitus en usuarios de quetiapina \\
\hline Koller ${ }^{17}$ & 2002 & Serie de casos de diabetes mellitus en usuarios de olanzapina \\
\hline Koller $^{18}$ & 2001 & Serie de casos de diabetes mellitus en usuarios de clozapina \\
\hline Kornegay ${ }^{19}$ & 2002 & No reporta el número de eventos \\
\hline Krosnick ${ }^{20}$ & 2005 & No excluyó pacientes con diabetes mellitus prevalente \\
\hline Leslie $^{21}$ & 2004 & No reporta el número de eventos \\
\hline Liebzeit 22 & 2001 & Revisión sistemática de series de casos \\
\hline Mackin 23 & 2005 & No excluyó pacientes con diabetes mellitus prevalente \\
\hline Meyer $^{24}$ & 2005 & Comparó antipsicóticos atípicos \\
\hline Sernyak $^{25}$ & 2005 & $\begin{array}{l}\text { Comparó antipsicóticos atípicos, no excluyó pacientes con diabetes } \\
\text { mellitus prevalente }\end{array}$ \\
\hline Sernyak $^{26}$ & 2003 & Desenlace fue hiperglicemia \\
\hline Taylor 27 & 2005 & No excluyó pacientes con diabetes mellitus prevalente \\
\hline Taylor 28 & 2004 & No excluyó pacientes con diabetes mellitus prevalente \\
\hline $\mathrm{Wu}^{29}$ & 2007 & No consideró diabetes mellitus como desenlace \\
\hline
\end{tabular}


Tabla 2. Estudios originales incluidos en el análisis y calidad de la evidencia

\begin{tabular}{|c|c|c|c|c|c|}
\hline ID Estudio & Diseño & Fuente & Exposiciones & Comentario & $\begin{array}{l}\text { Score } \\
0 \text { xford }\end{array}$ \\
\hline Bamer ${ }^{33}$ & $\begin{array}{l}\text { Cohorte } \\
\text { retrospectiva }\end{array}$ & $\begin{array}{l}\text { Base datos } \\
\text { Sistema } \\
\text { de Salud }\end{array}$ & $\begin{array}{l}\text { A. Convencionales } \\
\text { A. Atípicos }\end{array}$ & $\begin{array}{l}\text { Definición de } \\
\text { DM poco } \\
\text { sensible }\end{array}$ & 4 \\
\hline Buse $^{34}$ & $\begin{array}{l}\text { Cohorte } \\
\text { retrospectiva }\end{array}$ & $\begin{array}{l}\text { Base datos } \\
\text { farmacéutica }\end{array}$ & $\begin{array}{l}\text { A. Convencionales } \\
\text { A. Atípicos }\end{array}$ & $\begin{array}{c}\text { Definición de } \\
\text { DM poco sensible }\end{array}$ & 4 \\
\hline Cavazzoni35 & $\begin{array}{l}\text { Meta-análisis } \\
\text { de ensayos } \\
\text { clínicos }\end{array}$ & MEDLINE & $\begin{array}{l}\text { Placebo } \\
\text { Haloperidol } \\
\text { A. Atípicos }\end{array}$ & $\begin{array}{l}\text { Definición de } \\
\text { DM poco } \\
\text { sensible }\end{array}$ & $*$ \\
\hline Farwell ${ }^{36}$ & $\begin{array}{l}\text { Cohorte } \\
\text { retrospectiva }\end{array}$ & $\begin{array}{l}\text { Base datos } \\
\text { farmacéutica }\end{array}$ & $\begin{array}{l}\text { A. Convencionales } \\
\text { olanzapina } \\
\text { risperidona }\end{array}$ & $\begin{array}{l}\text { Definición de } \\
\text { DM poco } \\
\text { sensible }\end{array}$ & 4 \\
\hline Fuller ${ }^{37}$ & $\begin{array}{l}\text { Cohorte } \\
\text { retrospectiva }\end{array}$ & $\begin{array}{l}\text { Base datos } \\
\text { Sistema de } \\
\quad \text { Salud }\end{array}$ & $\begin{array}{l}\text { Haloperidol } \\
\text { Flufenazina } \\
\text { Olanzapina } \\
\text { Risperidona }\end{array}$ & $\begin{array}{l}\text { Definición de } \\
\text { DM poco } \\
\text { sensible }\end{array}$ & 4 \\
\hline Gianfrancesco ${ }^{38}$ & $\begin{array}{l}\text { Cohorte } \\
\text { retrospectiva }\end{array}$ & $\begin{array}{l}\text { Base datos } \\
\text { Sistema de } \\
\text { Salud }\end{array}$ & $\begin{array}{l}\text { A. Convencionales } \\
\text { Olanzapina } \\
\text { Risperidona }\end{array}$ & $\begin{array}{l}\text { Definición de } \\
\text { DM poco } \\
\text { sensible }\end{array}$ & 4 \\
\hline Koro $^{39}$ & $\begin{array}{l}\text { Caso/Control } \\
\text { anidado }\end{array}$ & $\begin{array}{l}\text { Base datos } \\
\text { Sistema de } \\
\text { Salud }\end{array}$ & $\begin{array}{l}\text { A. Convencionales } \\
\text { Olanzapina } \\
\text { Risperidona } \\
\text { Otros atípicos }\end{array}$ & $\begin{array}{l}\text { Definición de } \\
\text { DM poco } \\
\text { sensible }\end{array}$ & 4 \\
\hline Lund $^{40}$ & $\begin{array}{l}\text { Cohorte } \\
\text { retrospectiva }\end{array}$ & $\begin{array}{l}\text { Programa de } \\
\text { esquizofrenia }\end{array}$ & $\begin{array}{l}\text { A. Convencionales } \\
\text { Clozapina }\end{array}$ & $\begin{array}{c}\text { Definición de } \\
\text { DM poco sensible }\end{array}$ & 4 \\
\hline Miller ${ }^{41}$ & $\begin{array}{l}\text { Cohorte } \\
\text { retrospectiva }\end{array}$ & $\begin{array}{l}\text { Base datos } \\
\text { farmacéutica }\end{array}$ & $\begin{array}{l}\text { A. Convencionales } \\
\text { Clozapina } \\
\text { Olanzapina } \\
\text { Risperidona }\end{array}$ & $\begin{array}{l}\text { Definición de } \\
\text { DM poco } \\
\text { sensible }\end{array}$ & 4 \\
\hline Ollendorf ${ }^{42}$ & $\begin{array}{c}\text { Cohorte } \\
\text { retrospectiva }\end{array}$ & $\begin{array}{l}\text { Base datos } \\
\text { farmacéutica }\end{array}$ & $\begin{array}{l}\text { A. Convencionales } \\
\text { A. Atípicos }\end{array}$ & $\begin{array}{c}\text { Definición de } \\
\text { DM poco sensible }\end{array}$ & 4 \\
\hline Peuskens ${ }^{43}$ & Ensayo clínico & $\begin{array}{l}\text { Pacientes con } \\
\text { esquizofrenia }\end{array}$ & $\begin{array}{c}\text { Olanzapina } \\
\text { Amisulpiride }\end{array}$ & $\begin{array}{c}\text { Definición de } \\
\text { DM poco sensible }\end{array}$ & 4 \\
\hline Sernyak $^{44}$ & Corte transversal & $\begin{array}{l}\text { Base datos } \\
\text { Sistema de } \\
\text { Salud }\end{array}$ & $\begin{array}{l}\text { A. Convencionales } \\
\text { A. Atípicos }\end{array}$ & $\begin{array}{l}\text { Exclusión de } \\
\text { DM prevalente } \\
\text { poco sensible }\end{array}$ & 4 \\
\hline
\end{tabular}

DM: Diabetes Mellitus 2. *: No reporta homogeneidad.

prevalente e incidente fueron poco sensibles y en el caso de los estudios de cohortes la fuente de información fue principalmente registros farmacéuticos. El metaanálisis no reportó pruebas estadísticas de homogeneidad ni consistencia.

En la Figura 1, al comparar 62.903 usuarios de antipsicóticos atípicos con 36.383 usuarios de antipsi- cóticos convencionales, no encontramos diferencias significativas en el riesgo de diabetes mellitus 2 incidente (OR 1,02 IC 95\% 0,97 a 1,07 p 0,42). Al analizar por separado cada antipsicótico atípico (Figuras 2, 3, 4 y 5), la clozapina exhibió una tendencia no significativa a un mayor riesgo de diabetes mellitus 2 incidente (OR 1,30 IC 0,91 a 1,86), en tanto que el uso 


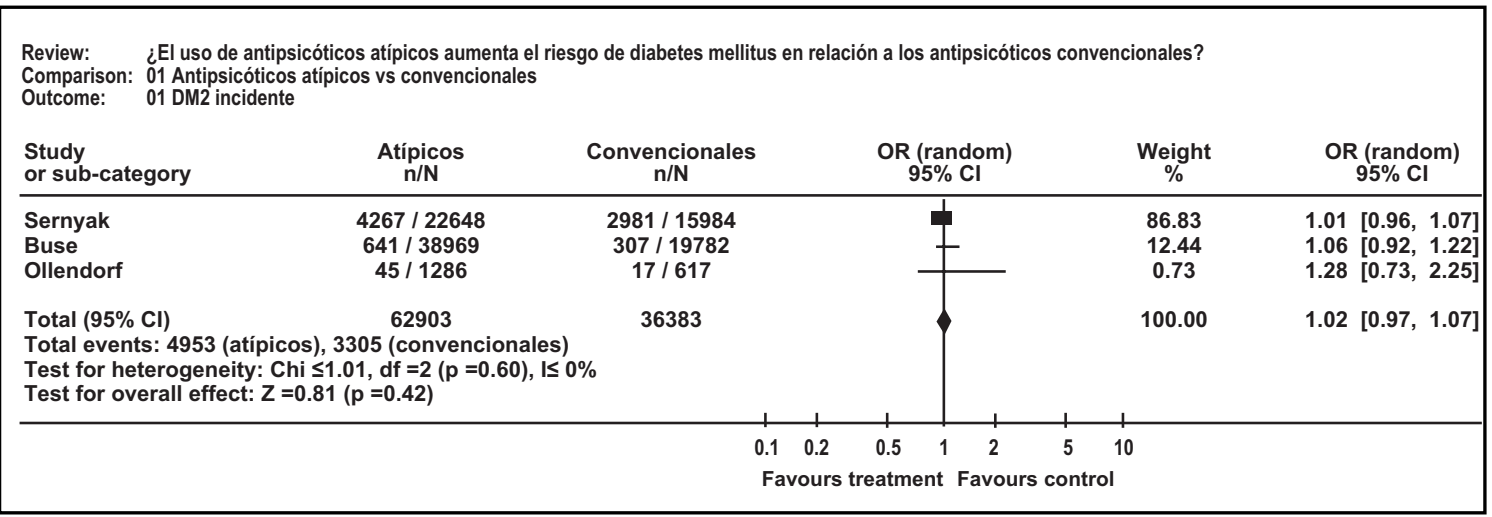

Figura 1. Magnitud del riesgo de diabetes mellitus 2 incidente, expresado como OR, en sujetos en tratamiento con antipsicóticos atípicos en relación con antipsicóticos convencionales.

\begin{tabular}{|c|c|c|c|c|c|}
\hline \multicolumn{6}{|c|}{$\begin{array}{l}\text { Review: ¿El uso de antipsicóticos atípicos aumenta el riesgo de diabetes mellitus en relación a los antipsicóticos convencionales? } \\
\text { Comparison: } 01 \text { Antipsicóticos atipicos vs convencionales } \\
\text { Outcome: } \\
02 \text { DM2 incidente }\end{array}$} \\
\hline $\begin{array}{l}\text { Study } \\
\text { or sub-category }\end{array}$ & $\underset{n / N}{C}$ & $\begin{array}{c}\text { Convencionales } \\
n / N\end{array}$ & $\begin{array}{l}\text { OR (random) } \\
95 \% \mathrm{Cl}\end{array}$ & $\begin{array}{c}\text { Weight } \\
\%\end{array}$ & $\begin{array}{l}\text { OR (random) } \\
95 \% \mathrm{CI}\end{array}$ \\
\hline Lund & $21 / 531$ & 78 / 2296 & - & 52.34 & $1.17[0.72,1.91]$ \\
\hline Buse & $7 / 277$ & 307 / 19786 & $\rightarrow$ & 21.95 & $1.64[0.77,3.51]$ \\
\hline Ollendorf & $2 / 35$ & $17 / 617$ & & 5.57 & $2.14[0.47,9.65]$ \\
\hline Miller & $7 / 84$ & $145 / 1981$ & & 20.15 & $1.15[0.52,2.54]$ \\
\hline \multirow{3}{*}{\multicolumn{3}{|c|}{$\begin{array}{l}\text { Total }(95 \% \mathrm{Cl}) \\
\text { Total events: } 37 \text { (Clozapina), } 547 \text { (convencionales) } \\
\text { Test for heterogeneity: Chi } \leq 1.06, \mathrm{df}=3(p=0.79), I \leq 0 \% \\
\text { Test for overall effect: } Z=1.45(p=0.15)\end{array}$}} & & 100.00 & $1.30[0.91,1.86]$ \\
\hline & & & & & \\
\hline & & & & & \\
\hline \multicolumn{6}{|c|}{$\begin{array}{lllllll}0.1 & 0.2 & 0.5 & 1 & 2 & 5 & 10\end{array}$} \\
\hline \multicolumn{6}{|c|}{ Favours treatment Favours control } \\
\hline
\end{tabular}

Figura 2. Magnitud del riesgo de diabetes mellitus 2 incidente, expresado como OR, en sujetos en tratamiento con clozapina en relación con antipsicóticos convencionales.

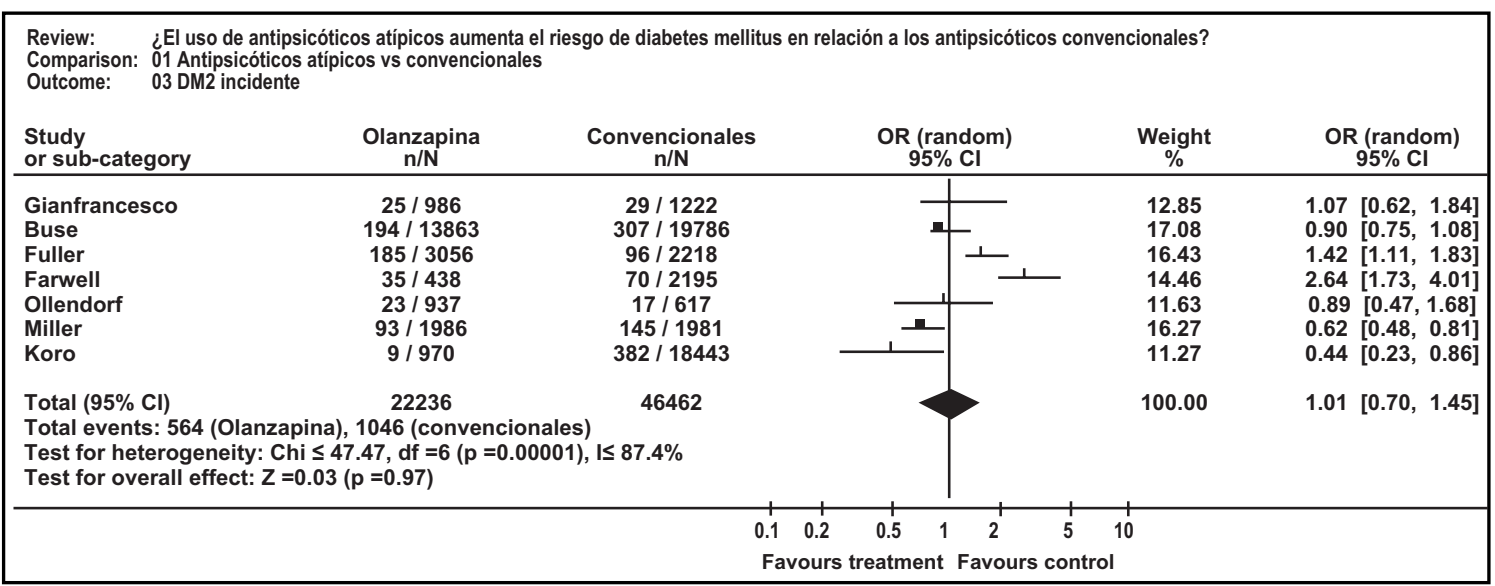

Figura 3. Magnitud del riesgo de diabetes mellitus 2 incidente, expresado como OR, en sujetos en tratamiento con olanzapina en relación con antipsicóticos convencionales. 


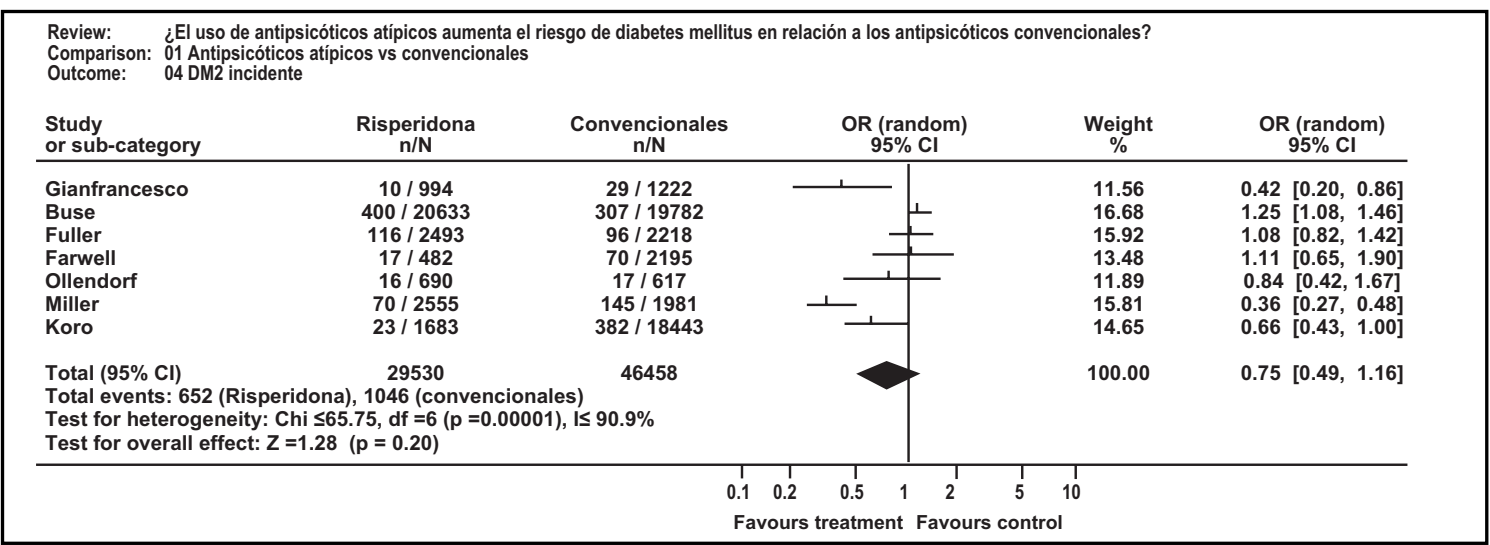

Figura 4. Magnitud del riesgo de diabetes mellitus 2 incidente, expresado como OR, en sujetos en tratamiento con risperidona en relación con antipsicóticos convencionales.

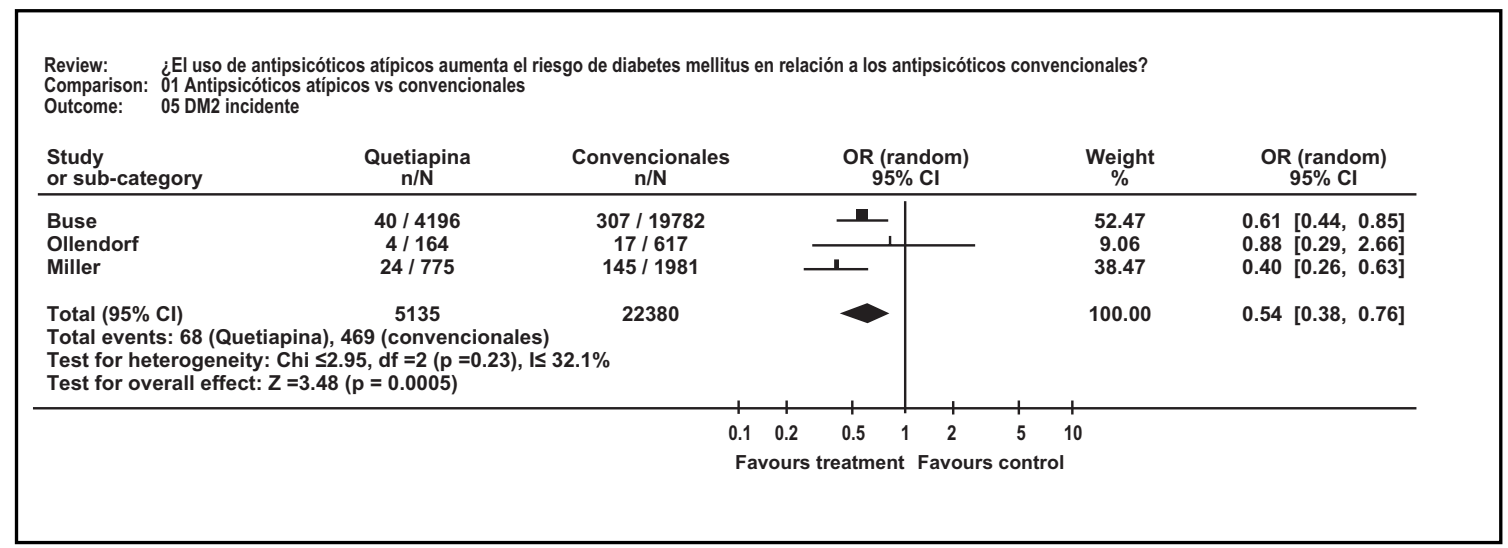

Figura 5. Magnitud del riesgo de diabetes mellitus 2 incidente, expresado como OR, en sujetos en tratamiento con quetiapina en relación con antipsicóticos convencionales.

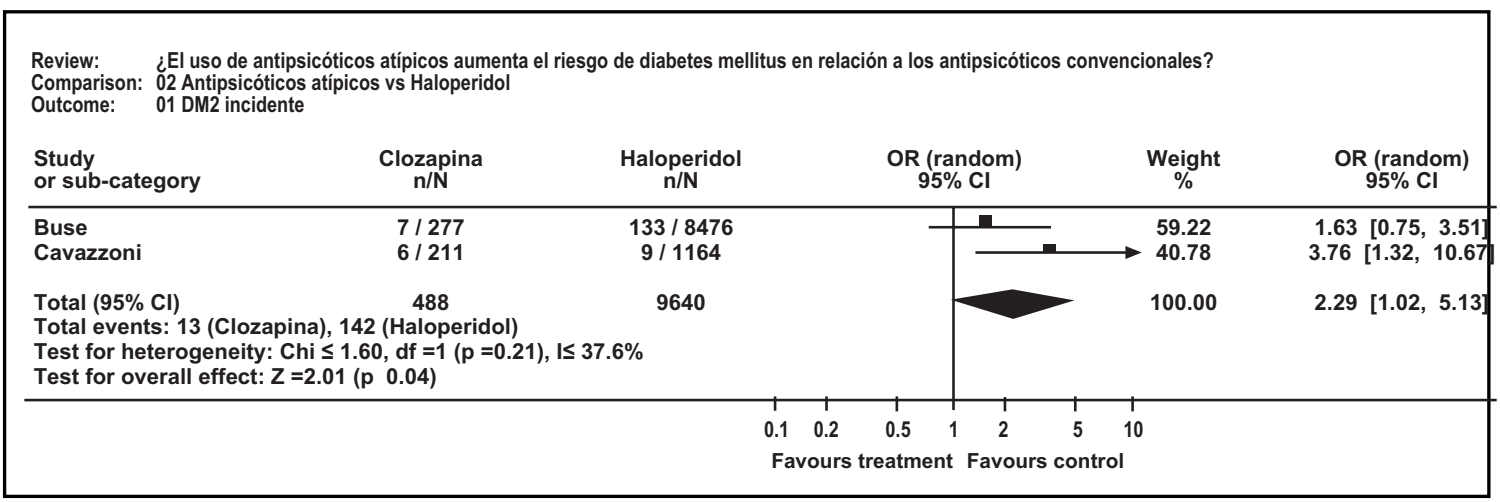

Figura 6. Magnitud del riesgo de diabetes mellitus 2 incidente, expresado como OR, en sujetos en tratamiento con clozapina en relación con haloperidol. 


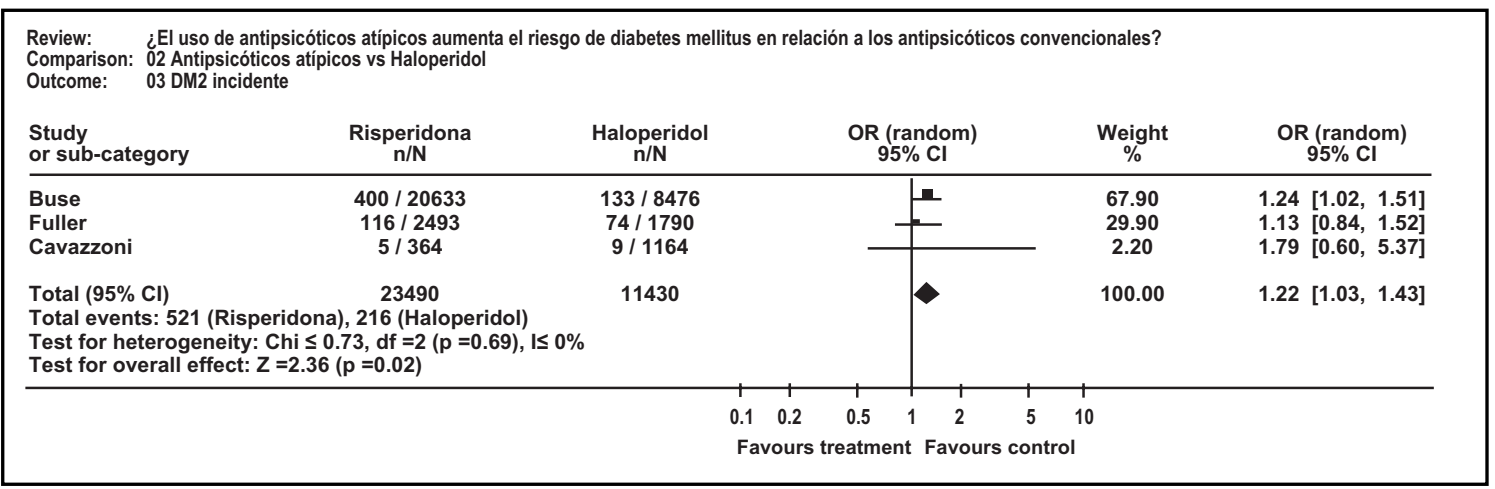

Figura 7. Magnitud del riesgo de diabetes mellitus 2 incidente, expresado como OR, en sujetos en tratamiento con risperidona en relación con haloperidol.

de quetiapina se asoció con menor riesgo que el de los antipsicóticos convencionales (OR 0,54 IC 95\% 0,41 a $0,69 \mathrm{p} \varangle 0,001$ ).

Al comparar los antipsicóticos atípicos con algunos antipsicóticos convencionales encontramos que el uso de clozapina se asoció con aumento significativo del riesgo de diabetes mellitus 2 incidente en relación al de haloperidol (Figura 6), efecto que también se observó con risperidona pero en menor magnitud (Figura 7). El resto de las comparaciones (quetiapina vs haloperidol, antipsicóticos atípicos versus tioridazina, amisulpiride, flufenazina o placebo) se fundamentan en datos sólo de un estudio original en cada caso. Sólo fue significativa la reducción en el riesgo de diabetes mellitus 2 incidente de la quetiapina en relación al haloperidol (OR 0,6 IC $95 \% 0,42$ a 0,86$)$.

\section{DisCUSIÓN}

Hace más de 40 años ya se había descrito que tratamientos prolongados con fenotiazinas de baja potencia se asociaban con aumento ponderal. A partir de la década de los noventa y con la incorporación de los antipsicóticos atípicos como fármacos de primera línea en el tratamiento de enfermedades psiquiátricas crónicas, este problema clínico adquirió importancia epidemiológica ${ }^{2}$. Sin embargo estudios poblacionales también han encontrado que la obesidad es más frecuente entre los pacientes con esquizofrenia y otras enfermedades psiquiátricas crónicas que la población general, independientemente de su tratamiento ${ }^{1,31}$.

Existen estudios en animales y humanos que sugieren que la administración de antipsicóticos atípicos inducen hiperfagia y disminuyen la saciedad. Sólo ziprasidona y aripiprazol no son agonistas de receptores $5 \mathrm{HT}_{1 \mathrm{a}}$ y $\mathrm{D}_{2}$, vinculados al control del apetito ${ }^{1,2}$.

Los efectos metabólicos de los antipsicóticos atípicos también han sido evaluados, destacando hiperinsulinismo y resistencia a la insulina demostradas con clozapina y olanzapina ${ }^{9,10}$. Chiu reportó que olanzapina reducía la respuesta insulínica a la hiperglicemia, sugiriendo que este fármaco también afectaba la función de las células $\aleph^{5}$. Hallazgos de significado incierto son una menor activación del eje hipotálamo-hipófisis-suprarrenales y aumento en los niveles de leptina con pérdida de la correlación entre los niveles de esta citoquina y el $\mathrm{IMC}^{1,2}$.

El efecto ponderal de los antipsicóticos atípicos en una población usuaria con riesgo cardiometabólico aumentado, ya sea por efectos en el apetito $o$ en la sensibilidad a la insulina necesariamente lleva a considerar un riesgo aumentado de diabetes mellitus 2. Existen múltiples reportes de casos clínicos de diabetes mellitus 2 incidente 0 agravamiento de diabetes mellitus prevalente con clozapina y olanzapina principalmente y con menor frecuencia para risperidona, quetiapina y ziprasidona ${ }^{1,17-19,23}$. Al seguir por 5 y 10 años una gran serie clínica de usuarios de clozapina se 
reportó una frecuencia de hiperglicemia de ayuno (>126 mg/dl) de 36,6\% y 43\%, respectivamente ${ }^{15,16}$.

El aumento en la incidencia de diabetes mellitus 2 asociado al uso de antipsicóticos atípicos es un efecto no deseado del tratamiento, por lo que en un ensayo clínico controlado sólo podría ser considerado como desenlace secundario o reportado como efecto adverso. Otra posibilidad es considerarla como una variable de respuesta en un estudio observacional de cohortes, diseño en que sería fundamental excluir de la mejor forma posible la diabetes mellitus prevalente y considerar variables de control que afecten el riesgo de diabetes mellitus incidente. Todos estos hechos permitían anticipar que la calidad de la evidencia disponible para responder la pregunta de búsqueda no sería óptima.

Once de los 12 estudios incluidos en nuestro análisis tenían un puntaje 4 en la escala de Oxford $\mathrm{y}$ todos utilizaron criterios para definir diabetes mellitus poco satisfactorios (uso de hipoglicemiantes orales o insulina, autorreporte, diagnóstico en la ficha clínica). En las 8 cohortes retrospectivas analizadas la fuente de información

\section{REFERENCIAS}

1. Ananth J, Parameswaran S, Gunatilake S. Side effects of atypical antipsychotic drugs. Current Pharmaceutical Design 2004; 10: 2219-29.

2. Baptista T, Zárate J, Joober R, Colosante C, Beaulieu S, PÁez X, Hernández L. Drug induced weight gain, and impediment to succesfull pharmacotherapy: Focus in antipsychotics. Current Drug Targets 2004; 5: 279-99.

3. ǗoK A, GAEBel W. Side effects of atypical antipsychotics: A brief overview. World Psychiatry 2008; 7: 58-62.

4. Andersen SW, Clemow DB, Corya SA. Long-term weight gain in patients treated with open-label olanzapine in combination with fluoxetine for major depressive disorder. J Clin Psychiatry 2005; 66: 1468-76.

5. Chiu C, Chen K, Lu H, Lu M. The early effect of Olanzapine and Risperidone on insulin secretion in atypical-näive schizophrenic patients. J Clin Psychopharmacol 2006; 26: 504-7. fueron registros de prescripción de fármacos y fichas clínicas de centros de salud. La única revisión sistemática disponible no reportó pruebas estadísticas para describir la homogeneidad de sus resultados.

Con todas estas limitaciones podemos concluir que el uso de clozapina se asoció con un importante aumento del riesgo de diabetes mellitus 2 incidente en comparación con el uso de haloperidol, efecto que también mostró risperidona pero en menor cuantía. El uso de quetiapina se asoció con un menor riesgo de diabetes mellitus incidente que el uso de antipsicóticos convencionales.

Es necesario diseñar mejores estudios para contestar la pregunta de búsqueda y que permitan identificar pacientes en riesgo de desarrollar la complicación.

\section{Agradecimientos}

Queremos agradecer la valiosa cooperación de la Sra. Cristina Miranda, del Centro Saval Temuco en la obtención de las referencias bibliográficas para el estudio.

6. Hardy TA, Meyers AL, Yu J, Shankar SS, Steinberg HO, PorsKen NK. Acute insulin response and B-cell compensation in normal subjects treated with Olanzapine or Risperidone for 2 weeks. Diabetes Care 2007; 30: 157-8.

7. Henderson DC, Cagliero E, Copeland PM, Borba CP, Evins E, HaYden D ET AL. Glucose metabolism in patients with schizophrenia treated with atypical antipsychotic agents. A frequently sampled intravenous glucose tolerance test and minimal model analysis. Arch Gen Psychiatry 2005; 62: 19-28.

8. Howes OD, Smith S, Gaughran FP, Amiel SA, Murray RM, PILOWsKY LS. The relationship between prolactin levels and glucose homeostasis in antipsychotictreated schizophrenic patients. J Clin Psychopharmacol 2006; 6: 629-31.

9. McKee JR, Bodfish JW, Mahorney SL, Heeth WL, BaLL MP. Metabolic effects associated with atypical antipsychotic treatment in the developmentally disabled. J Clin Psychiatry 2005; 66: 1161-8.

10. MeLKersson KI, DAHL ML. Relationship between levels of insulin or triglycerides and serum concentrations 
of the atypical antipsychotics clozapine and olanzapine in patients on treatment with therapeutic doses. Psychopharmacology 2003; 170: 157-66.

11. Wu R, Zhaо J, Zhai J, Guo X, Guo W. Sex difference in effects of typical and atypical antipsychotics on glucose-insulin homeostasis and lipid metabolism in first-episode schizophrtenia. J Clin Psychophamacol 2007; 27: 374-9.

12. Caro JJ, Ward A, Levinton C, Robinson K. The risk of diabetes during Olanzapine use compared with Risperidone use: A retrospective database analysis. J Clin Psychiatry 2002; 63: 1135-9.

13. De Hert M, Schreurs V, Sweers K, Van Eyck D, HANSSENS L, SinKo S et al. Typical and atypical antipsychotics differentially affect long-term incidence rates of the metabolic syndrome in firstepisode patients with schizophrenia: A retrospective chart review. Schizophrenia Research 2008; 101: 295-303.

14. Filakovic P, Laufer D, Radanovic-Grguric L, Koic O, FiJAcko M, DuRKovic M. Newer antipsychotics and glucose metabolism: A comparison between Olanzapine and Risperidone. Psychiatria Danubina 2005; 17: 63-6.

15. Henderson DC, Caglero E, Gray C, Nasralah RA, Hayden DL, Schoenfeld DA, Goff DC. Clozapine, diabetes mellitus, weight gain, and lipid abnormalities: A five-year naturalistic study. Am J Psychiatry 2000; 157: 975-81.

16. Henderson DC, Nguyen DD, Copeiand PM, Hayden DL, Borba CP, Louie PM et al. Clozapine, diabetes mellitus, hyperlipidemia, and cardiovascular risks and mortality: Results of a 10-year naturalistic study. J Clin Psychiatry 2005; 66: 1116-21.

17. Koler EA, Weber J, Doraiswamy PM, Schneider BS. A survey of reports of Quetiapine-associated hyperglycemia and diabetes mellitus. J Clin Psychiatry 2004; 65: 857-63

18. Koler E, Doraiswamy P. Olanzapine-associated diabetes mellitus. Pharmacotherapy 2002; 22: 841-52.

19. Koler E, Schneider B, Bennett K, Dubitsky G. Clozapine-associated diabetes. Am J Med 2001; 111: 716-23.

20. Kornegay CJ, Vasilakis-Scaramozza C, Jick H. Incidente diabetes associated with antipsychotic use in the United Kingdom General Practice Research Database. J Clin Psychiatry 2002; 63: 758-62.

21. KRosNicK A, WiLSON MG. A retrospective chart review of the clinical effects of atypical antipsychotic drugs on glycemic control in institutionalized patients with schizophrenia and comorbid diabetes mellitus. Clin Ther 2005; 27: 320-6.

22. LESLE DL, ROSENHECK RA. Incidence of newly diagnosed diabetes attribuible to atypical antipsychotic medications. Am J Psychiatry 2004; 161: 1709-11.

23. Liebzeit KA, Markowitz JS, Caley CF. New onset diabetes and atypical antipsychotics. European Neuropsychopharmacology 2001; 11: 25-32.

24. Mackin P, Watkinson HM, Young AH. Prevalence of obesity, glucose homeostasis disorders and metabolic syndrome in psychiatric patients taking typical or atypical antipsychotic drugs: A cross-sectional study. Diabetologia 2005; 48: 1430-1.

25. Meyer JM, Pandina G, Bossie CA, Turkoz I, Greenspan A. Effects of switching from Olanzapine to Risperidone on the prevalence of the metabolic syndrome in overweight or obese patients with schizophrenia or schizoaffective disorder: Analysis of a multicenter, rater-blinded, open-label study. Clin Ther 2005; 27: 1930-41.

26. SeRNYAK MJ, Gulanski B, Rosenheck R. Undiagnosed in patients treated with atypical antipsychotics. J Clin Psychiatry 2005; 66: 1463-7.

27. Sernyak MJ, Gulanski B, Lesle DL, Rosenheck R. Undiagnosed hyperglycemia in Clozapine-treated patients with schizophrenia. J Clin Psychiatry 2003; 64: 605-8.

28. Taylor D, Young C, Mohamed R, Paton C, Walwyn R. Undiagnosed impaired fasting glucose and diabetes mellitus among inpatients antipsychotic drugs. J Psychopharmacol 2005; 19: 182-6.

29. Taylor D, Young C, Esop R, Paton C, Walwyn R. Testing for diabetes in hospitalized patients prescribed antipsychotic drugs. BJP 2004; 185: 152-6.

30. HendeRson DC. Clinical experience with insulin resistance, diabetic ketoacidosis, and type 2 diabetes mellitus in patients treated with atypical antipsychotic agents. J Clin Psychiatry 2001; 62(suppl 27): 10-14.

31. Susce MT, Vilanueva N, Díaz FJ, de Lfón J. Obesity and associated complications in patients with severe mental illness: A cross-sectional survey. J Clin Psychiatry 2005; 66: 167-173.

32. Subramaniam M, Chong S, PeK E. Diabetes mellitus and impaired glucose tolerance in patients with schizophrenia. Can J Psychiatry 2003; 48: 345-7.

33. Barner JC, Worchel J, Yang M. Frequency of newonset diabetes mellitus and use of antipsychotic drugs among Central Texas veterans. Pharmacotherapy 2004; 24: 1529-38.

34. Buse JB, Cavazzoni P, Hornbuckle K, Hutchins D, BREIER A, Jovanovic L. A retrospective cohort study of diabetes mellitus and antipsychotic treatment in the United States. J Clin Epidemiol 2003; 56: 164-70.

35. Cavazzoni P, Mukhopadhyay N, Carlson C, Breier A, BUSE J. Retrospective analysis of risk factors in patients with treatment-emergent diabetes during clinical trials of antipsychotic medications. BJP 2005; 185 (suppl 47): s94-s101.

36. Farwell WR, Stump TE, Wang J, Tafesse E, L'Italien G, TiERNEY WM. Weight gain and new onset diabetes 
associated with Olanzapine and Risperidone. J Gen Intern Med 2004; 19: 1200-5.

37. Fuler MA, Shermock KM, Secic M, Grogg AL. Comparative study of the development of diabetes mellitus in patients taking Risperidone and Olanzapine. Pharmacotherapy 2003; 23: 1037-43.

38. Gianfrancesco FD, Grogg Al, Mahmoud RA, Wang R, NASRALAH HA. Differential effects of Risperidone, Olanzapine, Clozapine, and conventional antipsychotics on type 2 diabetes: Findings from a large health plan database. J Clin Psychiatry 2003; 63: 92030.

39. Koro CE, Fedder DO, L'Italen G, Weiss SS, Magder LS, KreyenBuhl J et aL. Assessment of independent effect of Olanzapine and Risperidone on risk of diabetes among patients with schizophrenia: population based nested case-control study. BMJ 2002; 325: 243-7.

40. Lund BC, Perry PJ, Brooks JM, Arndt S. Clozapine use in patients with schizophrenia and the risk of diabetes, hyperlipidemia, and hypertension. Arch Gen Psychiatry 2001; 58: 1172-6.

41. Muler EA, Leslie DL, Rosenheck RA. Incidence of new-onset diabetes mellitus among patients receiving atypical neuroleptics in the treatment of mental illness. J Nerv Ment Dis 2005; 193: 387-95.

42. OLendorf DA, Joyce AT, Rucker M. Rate of new-onset diabetes among patients treated with atypical versus conventional antipsychotic medications for schizophrenia. Med Gen Med 2004; 6: 5-13.

43. Peuskens J, de Hert M, Mortimer A. Metabolic control in patients with schizophrenia treated with amisulpiride or olanzapine. Int Clin Psychopharmacol 2007; 22: 145-52.

44. Sernyak MJ, Leslie DL, Alarcón RD, Losonczy MF, ROSENHECK R. Association of diabetes mellitus with use os atypical neuroleptics in the treatment of schizophrenia. Am J Psychiatry 2002; 159: 561-6. 